\title{
casanova encodes a novel Sox-related protein necessary and sufficient for early endoderm formation in zebrafish
}

\author{
Yutaka Kikuchi, ${ }^{1,4}$ Antoine Agathon, ${ }^{2,4}$ Jonathan Alexander, ${ }^{1}$ Christine Thisse, ${ }^{2}$ Steven Waldron, ${ }^{1}$ \\ Deborah Yelon, ${ }^{1,3}$ Bernard Thisse, ${ }^{2}$ and Didier Y.R. Stainier ${ }^{1,5}$ \\ ${ }^{1}$ Department of Biochemistry and Biophysics, Programs in Genetics, Human Genetics, and Developmental Biology, \\ University of California, San Francisco, California 94143-0448, USA; ${ }^{2}$ Institut de Génétique et Biologie Moléculaire et \\ Cellulaire CNRS/INSERM/ULP, BP 163, 67404 Illkirch cedex, CU de Strasbourg, France
}

Early endoderm formation in zebrafish requires at least three loci that function downstream of Nodal signaling but upstream of the early endodermal marker sox17: bonnie and clyde (bon), faust (fau), and casanova (cas). cas mutants show the most severe phenotype as they do not form any gut tissue and lack all sox17 expression. Activation of the Nodal signaling pathway or overexpression of Bon or Fau/Gata5 fails to restore any sox17 expression in cas mutants, demonstrating that cas plays a central role in endoderm formation. Here we show that cas encodes a novel member of the Sox family of transcription factors. Initial cas expression appears in the dorsal yolk syncytial layer (YSL) in the early blastula, and is independent of Nodal signaling. In contrast, endodermal expression of cas, which begins in the late blastula, is regulated by Nodal signaling. Cas is a potent inducer of sox17 expression in wild-type embryos as well as in bon and fau/gata5 mutants. Cas is also a potent inducer of sox17 expression in MZoep mutants, which cannot respond to Nodal signaling. In addition, ectopic expression of cas in presumptive mesodermal cells leads to their transfating into endoderm. Altogether, these data indicate that Cas is the principal transcriptional effector of Nodal signaling during zebrafish endoderm formation.

[Key Words: Sox; gut endoderm; Nodal; bonnie and clyde; faust; casanova]

Received February 28, 2001; revised version accepted April 13, 2001.

Vertebrate endoderm formation has recently been the focus of intense investigations. In the late blastula stage zebrafish embryo, endodermal cells form in the four most marginal blastomere tiers (Warga and NüssleinVolhard 1999), and involute early during gastrulation to occupy the deep hypoblast, directly overlying the extraembryonic yolk syncytial layer (YSL; Warga and Kimmel 1990). During gastrulation, the endodermal cells are recognizable by their characteristically large size and flat morphology, distinct from the smaller, rounder mesodermal cells (Warga and Nüsslein-Volhard 1999). Starting at the onset of gastrulation, endodermal cells express sox17, a high-mobility group (HMG) domain transcription factor gene (Alexander and Stainier 1999), as well as foxA2/axial/HNF3 $\beta$, a winged helix/forkhead transcription factor gene (Strähle et al. 1993; Schier et al. 1997; Kaestner et al. 2000).

\footnotetext{
${ }^{3}$ Present address: Skirball Institute of Biomolecular Medicine, New York University School of Medicine, New York, NY 10016, USA.

${ }^{4}$ These authors contributed equally to this work.

${ }^{5}$ Corresponding author.

E-MAIL didier_stainier@biochem.ucsf.edu; FAX (415) 476-3892. Article and publication are at www.genesdev.org/cgi/doi/10.1101/ $\operatorname{gad} .892301$.
}

Several zebrafish mutations affect endoderm formation and endodermal sox17 and foxA2 expression. The earliest acting mutations, cyclops (cyc), squint (sqt), and oep, affect Nodal signaling: Cyc and Sqt are themselves Nodal-related proteins (Erter et al. 1998; Feldman et al. 1998; Rebagliati et al. 1998a; Sampath et al. 1998), and Oep is a transmembrane protein essential for Nodal signaling (Zhang et al. 1998; Gritsman et al. 1999). Embryos mutant for both $c y c$ and $s q t$, or embryos that lack both maternal and zygotic Oep (MZoep), fail to form all endoderm and most mesoderm (Feldman et al. 1998; Gritsman et al. 1999). casanova (cas), faust (fau), and bonnie and clyde (bon) more specifically affect endoderm formation and endodermal sox17 and foxA2 expression. The cas phenotype is the most dramatic, in that cas mutants do not form any gut tissue and fail to express any markers of endoderm differentiation or formation from the onset of gastrulation (Alexander et al. 1999; Alexander and Stainier, 1999).

In contrast to cas mutants that completely lack sox17 expression, fau and bon mutants exhibit a significant level; at bud stage, fau and bon mutants contain $\sim 60 \%$ and $10 \%$ of the wild-type number of sox17-expressing endodermal cells, respectively. fau, which encodes the 
zinc finger transcription factor Gata5 (Reiter et al. 1999), and bon, which encodes a Mix-type homeodomain protein (Kikuchi et al. 2000), are both expressed in the marginal domain of the embryo beginning at dome stage, that is, $\sim 80 \mathrm{~min}$ before the endodermal cells initiate sox17 and foxA2 expression (Alexander et al. 1999; Reiter et al. 1999,2001; Rodaway et al. 1999). The expression of both bon and fau/gata5 is regulated by Nodal signaling (Alexander and Stainier 1999; Rodaway et al. 1999; Reiter et al. 2001). And whereas bon expression ceases soon after gastrulation commences (Alexander et al. 1999|, fau/gata5 expression persists and is involved in maintaining endodermal sox17 and foxA2 expression during gastrulation (Reiter et al. 2001).

cas also functions downstream of Nodal signaling as indicated by the failure of a constitutively active form of the type-I transforming growth factor $\beta$ (TGF- $\beta$ ) receptor Taram-a (Renucci et al. 1996), a probable zebrafish ortholog of mammalian Alk4 (Payne et al. 2001) and Nodal receptor, to induce sox17 expression in cas mutants (Alexander and Stainier 1999). Overexpression of bon in cas mutants also fails to restore endodermal sox17 expression (Alexander and Stainier 1999), and similar data were obtained when fau/gata5 was overexpressed in cas mutants (Reiter et al. 2001). In addition, mosaic analysis indicates that cas acts cell-autonomously in the endodermal progenitors (Alexander et al. 1999).

These and other data have suggested the following pathway for zebrafish endoderm formation: Nodal signaling induces the expression of bon and fau/gata5; Bon and Fau/Gata5 cooperatively regulate the expression of sox17 and foxA2, but do not regulate each other's expression; Cas is essential cell autonomously for sox17 expression and appears to function downstream of, or in parallel to, Bon and Fau/Gata5 (Alexander and Stainier 1999; Kikuchi et al. 2000; Reiter et al. 2001). Clearly, many issues remain to be resolved regarding this pathway and the isolation of cas should help address some of them.

Studies in Xenopus also implicate Nodal-related molecules (Osada and Wright 1999), a Mix-type homeodomain protein, Mixer (Henry and Melton 1998), and Gata5 (Weber et al. 2000) in the regulation of Xsox17 expression and endoderm formation (Xanthos et al. 2001). XSox17 itself appears to be an important intrinsic regulator of endoderm formation; when overexpressed in animal caps, it activates endodermal gene expression, and overexpression of a dominant interfering variant of itself (Xsox17-enR) inhibits endoderm differentiation in both isolated vegetal pole explants and the intact frog embryo (Hudson et al. 1997).

The striking parallels between the pathways regulating endoderm formation in zebrafish and Xenopus suggest that similar molecules are likely to function in this process in mammals. Indeed, Nodal signaling has also been implicated in mouse endoderm development (Conlon et al. 1994; Tremblay et al. 2000), and Gata factors are expressed and function in a variety of developing tissues including the endoderm (for review, see Zaret 1999).

Given its central role in zebrafish endoderm forma- tion, we sought to isolate cas using a combined approach of positional cloning and candidate gene testing. Here, we show that cas encodes a novel member of the Sox family of HMG domain transcription factors. cas expression initiates in the dorsal YSL in the early blastula and is later found in the presumptive endodermal progenitors prior to their involution and initiation of sox17 expression. Cas is a potent inducer of sox17 expression in wildtype embryos, as well as in bon, fau/gata5, and MZoep mutants. In addition, ectopic expression of cas in mesodermal cells leads to their transfating into endodermal cells. These data illustrate the unique potency of Cas as a transcriptional regulator of endoderm formation in zebrafish.

\section{Results}

\section{Isolation of cas}

We initially mapped cas $^{\text {ta56 }}$ within $2 \mathrm{cM}$ of the LG7 centromere using half-tetrad analysis (Johnson et al. 1995): 94 wild-type and 88 mutant embryos were generated after blocking the second meiotic division of gynogenotes. Fine mapping revealed that cas is tightly linked to the simple sequence repeat markers Z7958 (no recombination in 5405 meiotic events) and Z9869 (no recombination in 550 meiotic events). We used these markers to begin to assemble a contiguous stretch of genomic DNA with yeast artificial chromosome and P1-derived artificial chromosome clones. However, it became clear that this region was underrepresented in the existing largeinsert genomic libraries (J. Alexander, S. Waldron, and D.Y.R. Stainier, unpubl.).

In the course of a large-scale, in situ hybridization screen for zebrafish genes expressed in restricted patterns during embryogenesis (C. Thisse and B. Thisse, unpubl.), we isolated CG569, a Sox-related gene expressed in the YSL and endodermal cells during gastrulation. In light of this expression pattern, we placed CG569 on the Goodfellow radiation hybrid ( $\mathrm{RH}$ ) panel (Kwok et al. 1999), and found that it mapped in the cas region (data not shown), suggesting that CG569 could correspond to cas. The CG569 genomic locus was characterized and sequenced in the wild-type and cas $^{\text {ta56 }}$ alleles. In cas $^{\text {ta56 }}$ we found a T-to-G substitution that introduces a premature translational stop codon at position 170 and leads to a truncation of the protein shortly after the HMG domain (Fig. 1A). During a mosaic F1 screen for cardiac mutations (Alexander et al. 1998), we identified a second mutant allele of cas, designated $\mathrm{cas}^{s 4}$. The phenotype of $\mathrm{cas}^{s 4}$ is indistinguishable from that of cas $^{\text {ta56 }}$, except that $\operatorname{cas}^{\text {s4 }}$ mutants exhibit some endodermal foxA2 expression that is not maintained during gastrulation (data not shown). In $\operatorname{cas}^{s 4}$ we found an A-to-G substitution that introduces an arginine in place of a highly conserved histidine at codon 130 within the HMG domain (Fig. 1A). An HMG domain has a "twisted letter L" or "boomerang" shape, and this histidine (His-65 within the HMG domain) is believed to contribute to the stability of the long arm of the $\mathrm{L}$, which is 
important for DNA binding and bending (Werner et al. 1995).

Using restriction fragment length polymorphisms to analyze linkage, we found that the ta56 and $s 4$ mutant alleles of CG569 segregated with the cas phenotype in 240 and 200 meiotic events, respectively (data not shown). Together with the tight genetic linkage between cas and Z7958, and the significant physical linkage between Z7958 and CG569 on the RH map, these data indicate that CG569 is very closely linked to cas. To test further whether the cas phenotype is caused by the identified mutations in CG569, we injected wild-type CG569 mRNA (100 pg) into embryos derived from cas $^{\text {ta56 }}$ heterozygote intercrosses, and genotyped the injected embryos following wholemount in situ hybridization with sox17. We found that this mRNA could restore sox17 expression in cas $^{\text {ta56 }}$ mutants (Fig. 1B), further suggesting that CG569 encodes Cas. We also confirmed that the s4 mutation inactivates Cas function by showing that $\operatorname{cas}^{s 4}$ mRNA injected into wild-type embryos had no effect on sox17 expression (data not shown). Considered together, the linkage data, identification of severe molecular lesions, and rescue experiments indicate that CG569 is cas.

Interestingly, we found that cas overexpression led to confluent patches of sox17 expression in both wild-type and cas mutant embryos (Figs. 1B, 4B, see below), in contrast to the regularly spaced distribution of sox17expressing endodermal cells observed in uninjected wildtype embryos (Fig. 4A, see below; Alexander and Stainier 1999). These data suggest that cas is able to activate sox17 expression in nonendodermal cells, and possibly direct cells not fated to become endoderm into the endodermal lineage. This hypothesis will be directly tested and the data shown in Figure 6 (below).

Sequence and clustering analyses indicate that Cas encodes a novel Sox protein of the SoxF group, which includes Sox7, Sox17, and Sox18 (Fig. 1C). Within the HMG domain, Cas shows less sequence identity $(<71 \%)$ with any of the existing SoxF members than the other SoxF members do with each other (>83\%) (Fig. 1D,E). (Contributing to this divergence are substitutions in the RPMNAFMVW signature motif common to the HMG domains of all non-SRY Sox proteins thus far identified [Bowles et al. 2000].) However, Cas shows a higher degree of sequence identity to the SoxF group than to the neighboring SoxE group; for example, Cas and mouse Sox10, a group E member, show only 54\% sequence identity in their HMG domains. Thus, considering all currently available data, Cas appears to be a novel member of the SoxF group.

\section{Regulation of cas expression}

cas expression is first observed at high stage $(3.3 \mathrm{~h}$ postfertilization [hpf]) in the dorsal YSL (Fig. 2A, arrowhead, B). Embryonic cas expression initiates $<1 \mathrm{~h}$ later in the dorsal marginal blastomeres (Fig. 2C,D), by which time cas is expressed at low levels throughout the YSL. The embryonic expression extends progressively around the margin, and by $40 \%$ epiboly ( $5 \mathrm{hpf}$ ) cas is clearly expressed all around the margin in both the YSL and marginal blastodermal cells (Fig. 2E). A high magnification view clearly shows cas expression in both the YSL, distinguished by the large nuclei, and the presumptive endodermal progenitors at the margin (Fig. 2F), whereas adjacent marginal cells, presumably mesodermal progenitors, do not express cas. Embryonic cas expression precedes sox17 expression and can be clearly seen during gastrulation, both in the endodermal cells as well as in the forerunner cells (Fig. 2G, fo; I-L), a specialized subset of dorsal cells that also express sox17 (Alexander and Stainier 1999) and sqt (Fig. 2L; Erter et al. 1998; Feldman et al. 1998; Rebagliati et al. 1998a), and later form the lining of Kupffer's vesicle (Cooper and D'Amico 1996). By $90 \%$ epiboly, cas expression is evident throughout the endoderm (Fig. 2G,H); posterior endodermal cells that express sox 17 but not foxA2 (Alexander and Stainier 1999) also express cas. At this stage cas expression is still present in the YSL, but it disappears shortly afterwards from the YSL as well as from embryonic tissues (data not shown).

Next, we examined whether cas expression is regulated by Nodal signaling. Embryos injected with 100 pg of mRNA encoding a constitutively active form of the type I TGF $\beta$ receptor Taram-a, designated Taram-a*, showed a massive induction of cas (Fig. 2N,O), indicating that Nodal signaling activates cas expression. Conversely, injection of $25 \mathrm{pg}$ of mRNA encoding Antivin, a competitive inhibitor of the Activin/Nodal signaling pathway related to mammalian Lefty (Thisse and Thisse 1999; Thisse et al. 2000), led to a complete loss of cas expression in marginal blastomeres at late blastula stage, whereas cas expression in the YSL appeared to be unaffected (Fig. 2P). Examining antivin-injected embryos at $90 \%$ epiboly confirmed the loss of cas expression in the endodermal and forerunner cells, but not the YSL (Fig. 2Q). Similarly, MZoep mutants exhibit no cas expression in the embryo, but seemingly normal cas expression in the YSL (data not shown). These data demonstrate that the embryonic expression of cas, like that of bon and fau/gata5, is regulated by Nodal signaling, whereas the YSL expression of cas is independent of Nodal signaling.

We then used embryonic cas expression as a marker of endoderm to examine endoderm formation in cas, bon, and fau/gata 5 mutants at shield ( $6 \mathrm{hpf})$ and $80 \%$ epiboly (8.3 hpf) stages. In cas mutants, no embryonic cas expression is observed, whereas cas expression appears to be up-regulated in the YSL (Fig. 3A,D). In bon and fau/ gata5 mutants, embryonic cas expression behaves essentially like sox17 expression. bon mutants exhibit a profound reduction in the number of cas-expressing endodermal cells but normal cas expression in the forerunners and YSL (Fig. 3B,E). fau/gata5 mutants show a smaller, though noticeable, decrease in the number of cas-expressing endodermal cells, as well as a decrease in the intensity of cas expression in the endodermal cells, whereas cas expression appears to be normal in the forerunners and YSL (Fig. 3C,F). These data further support 
Kikuchi et al.

\section{A}

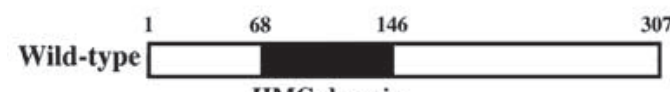

HMG domain
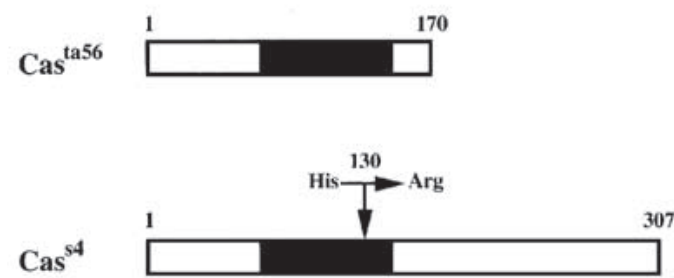

B

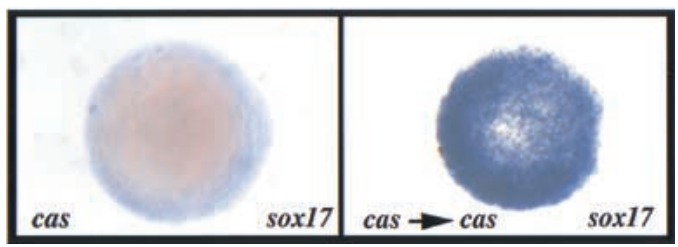

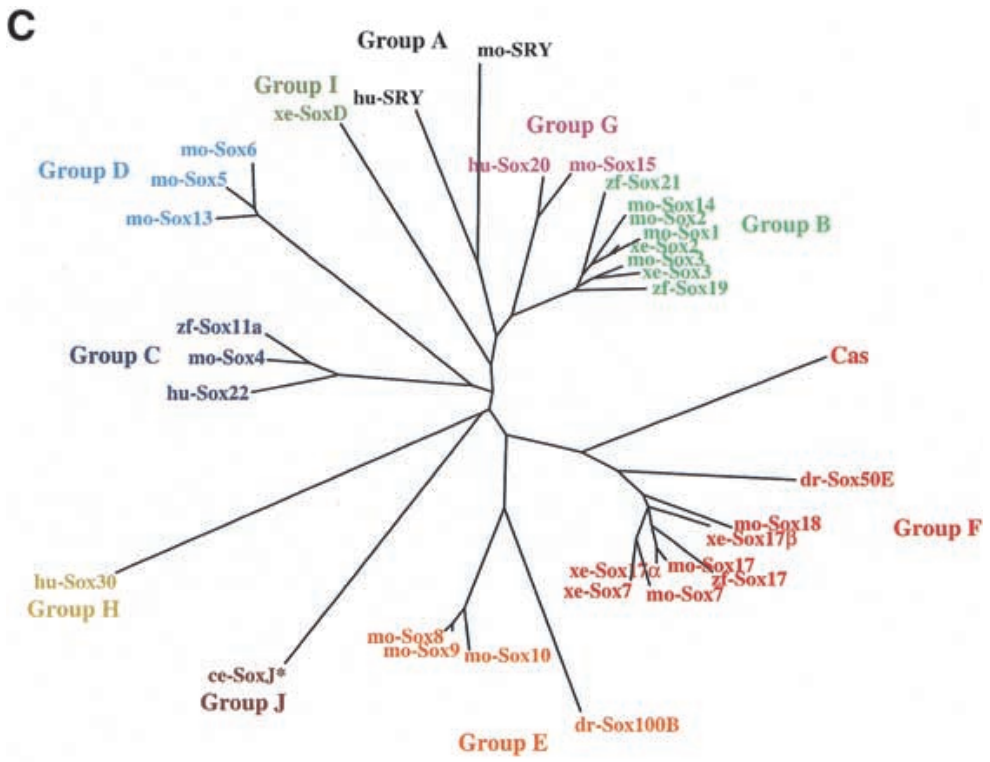

0.1

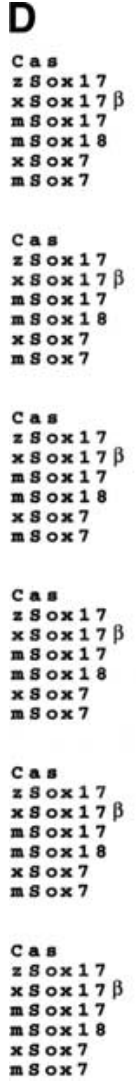

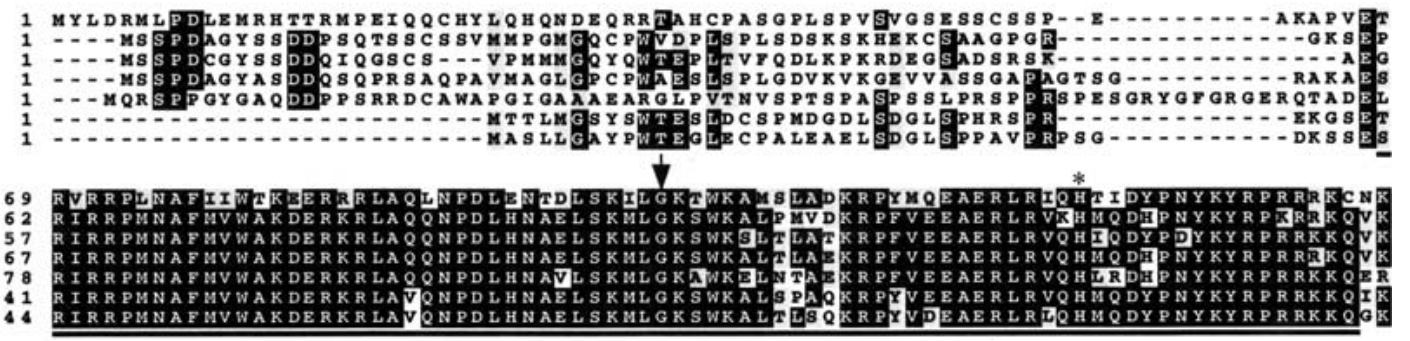
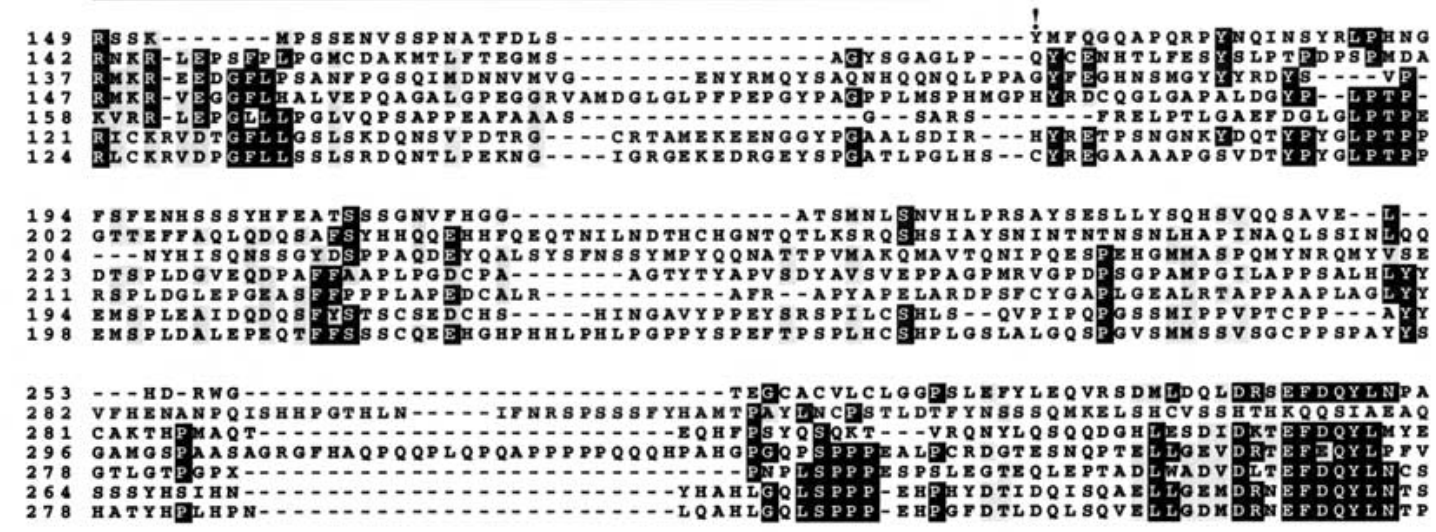

300 Q

357 SQASTATHSSGQMVDEVEYEHCLSFGVPSAPLPGSDLISTVLSDAS- SAVYYCGYNNS-

376 Y328 R 32 - TRPDATTLPYHVXLAKLGPRAMSCPE- - ESSLISALSDAS SAVYYSACISG 317
332

(Figure 1 continued on facing page) the hypothesis that no endoderm forms in cas mutants, and also further illustrate the close correspondence between cas and sox17 expression in the endoderm and forerunner cells (Alexander and Stainier 1999; Kikuchi et al. 2000; Reiter et al. 2001). cas can induce cyc and sqt expression

cas overexpression in wild-type embryos induces widespread sox17 expression (Fig. 4A,B), and inhibits epiboly and involution (data not shown). These phenotypes are 


\begin{tabular}{ccc}
\hline & \multicolumn{2}{c}{ Identity of HMG domain (\%) } \\
\cline { 2 - 3 } & Cas & zf-Sox17 \\
\hline zf-Sox17 & 65 & - \\
xe-Sox17 & 66 & 85 \\
mo-Sox17 & 68 & 91 \\
mo-Sox18 & 65 & 84 \\
xe-Sox7 & 70 & 86 \\
mo-Sox7 & 67 & 84 \\
mo-Sox10 & 54 & 66 \\
mo-LEF1 & 29 & 30 \\
\hline
\end{tabular}

Figure 1. cas encodes a novel member of the Sox family of high-mobility group (HMG) domain transcription factors. (A) Schematic representation of wild-type and mutant Cas proteins. The cas $^{\text {ta56 }}$ mutation leads to a truncation of the protein shortly after the HMG domain, and the $\mathrm{cas}^{\text {s4 }}$ mutation introduces a single amino acid substitution (histidine to arginine) in the third helix of the HMG domain (position 130). (B) sox17 expression in uninjected (left) and CG569/cas-injected cas $^{\text {ta56 }}$ mutant embryos. Animal pole views at shield stage $(6 \mathrm{~h}$ postfertilization) with dorsal to the right (left embryo). Whereas sox17 expression is missing in uninjected cas $^{\text {ta56 }}$ mutants (left), overexpression of CG569/cas mRNA always induced sox17 expression (right). For comparison to wild-type pattern of sox17 expression, see Fig. 4A. $(C)$ Unrooted phylogenetic tree for the Sox HMG domain, constructed by the neighbor joining (NJ) method (Saitou and Nei 1987). Branch lengths are representative of the extent of divergence. Ten groups (Groups A-J) are classified by phylogenetic analysis of Sox protein HMG domain sequences (Bowles et al. 2000). The ce-SoxJ ${ }^{\star}$ protein (Group J) was identified from databases and previously reported as a Sox protein (Bowles et al. 2000). This phylogenetic analysis indicates that Cas is a divergent member of the SoxF group. $(D)$ Sequence comparison of Cas; zebrafish, Xenopus and mouse Sox17; mouse Sox18; and Xenopus and mouse Sox7. The HMG domain is underlined. Cas shows a relatively high degree of sequence identity in its HMG domain with the members of the SoxF group, but otherwise shows limited sequence conservation. The arrow indicates intron position in cas, zebrafish sox17, and mouse Sox17 and Sox18, which appears to be the same in all members of the SoxF group but different in most other Sox genes (Bowles et al. 2000). The asterisk and exclamation point indicate the position of the $\operatorname{cas}^{s 4}$ and $\operatorname{cas}^{\text {ta56 }}$ mutations, respectively. (E) Percent sequence identity of the Cas and zebrafish Sox17 HMG domains with the HMG domains of the other members of the SoxF group as well as with the HMG domains of mouse Sox10 (SoxE group) and mouse LEF1. Cas shows substantially less sequence identity with the other SoxF group members than does zebrafish Sox17. However, Cas shows even less identity with mouse Sox10 (54\%) and mouse LEF1 (29\%). Abbreviations: ce, Caenorhabditis elegans; dr, Drosophila melanogaster; hu, Homo sapiens; mo, mouse; xe, Xenopus laevis; zf, Danio rerio.

very similar to those seen following taram- $a^{\star}$ overexpression (Alexander and Stainier 1999). Although embryonic cas expression and function are clearly downstream of Nodal signaling (Fig. 2N-Q; Alexander and Stainier
1999|, it seemed possible that cas overexpression could act by inducing the expression of $c y c$ and/or sqt. To investigate this possibility, we overexpressed cas (100 pg) in wild-type embryos at the 1-4-cell stage and examined $c y c$ and $s q t$ expression. We observed a significant increase in cyc and sqt expression (Fig. 4C-F), indicating that cas can activate their expression. However, except for the lack of sqt expression in the forerunners, which are missing in cas mutants (Alexander et al. 1999), we did not observe overt defects in cyc or sqt expression in cas mutants by wholemount in situ hybridization (data not shown).

Given that cas can activate $c y c$ and sqt expression, it seemed that cas could induce sox17 expression directly or via Nodal induction. To distinguish between these possibilities, we overexpressed cas in MZoep mutants, which cannot respond to Nodal signaling (Gritsman et al. 1999). We found that cas could induce sox17 expression in MZoep mutants (Fig. 4G,H), extending our previous data that Cas functions downstream of Nodal signaling (Alexander and Stainier 1999) by showing that it is sufficient to induce sox17 expression in the absence of Nodal signaling. In addition, we found that cas overexpression in MZoep mutants did not induce cyc or sqt expression (data not shown), indicating that this aspect of Cas function is dependent on Nodal signaling. Interestingly, cas-overexpressing MZoep mutants exhibited only faint foxA2 expression and no bon, fau/gata5, gsc, or $n t l$ expression (data not shown), suggesting that Cas cannot activate the expression of either bon or gata5, nor does it induce the expression of mesodermal markers. Rather, Cas can regulate sox17 expression in a Nodalindependent manner, illustrating its potency as an inducer of endodermal gene expression.

\section{Relationship of Cas, Bon and Fau/Gata5}

fau/gata5, bon, and cas are all induced by Nodal signaling, and each regulates sox17 expression (Alexander and Stainier 1999; Rodaway et al. 1999; Kikuchi et al. 2000; Reiter et al. 2001). Our previous work had shown that bon or fau/gata5 overexpression in cas mutants failed to restore sox17 expression (Alexander and Stainier 1999; Reiter et al. 2001), indicating that cas function is required downstream of bon and fau/gata5. To test this model further, we injected cas into bon and fau/gata5 mutants and examined sox17 expression. cas was able to expand sox17 expression in bon and fau/gata5 mutants to an extent comparable to that seen in wild-type embryos (Fig. 5A-C), confirming that cas is able to function in the absence of bon or fau/gata5.

Although Cas function clearly acts downstream of Bon and Fau/Gata5, the precise relationship between these proteins remained unclear. It could be that Nodal signaling induces bon and fau/gata5, which then activate cas, which directs endoderm formation. Alternatively, bon, fau/gata5, and cas could each be induced independently by Nodal signaling, and each could direct endoderm formation, with cas being uniquely required for sox17 ex- 
Figure 2. cas expression in zebrafish embryos at high (3.3 h postfertilization [hpf]; $A, B)$, sphere ( 4 hpf; $C, D), 30 \%$ epiboly $(4.7$ hpf; I), 40\% epiboly (5 hpf; E,F,N,O,P), $50 \%$ epiboly $(5.3 \mathrm{hpf} ; J), 60 \%$ epiboly $(7$ hpf; $K, L$ ), and $90 \%$ epiboly (9 hpf; G,H,Q) stages. sqt expression at $60 \%$ epiboly 17 hpf; M). A, C, E, F, G, L, M, N, P, and Q show lateral views with dorsal to the right. $B, D$, and $O$ show animal pole views. $H, I$, $I$, and $K$ show dorsal views with anterior to the top. $(A, B)$ cas expression is first observed at the high stage in the dorsal YSL. $(C, D)$ By sphere stage cas expression is detected throughout the marginal (yolk syncytial layer) YSL and in dorsal blastomeres. (E,F) At $40 \%$ epiboly cas is expressed in a subset of blastomeres all around the margin (see high magnification in $F$ ), and is maintained in the marginal YSL. $(G, H)$ As endodermal cells gastrulate, they continue to express cas, and a group of noninvoluting cells, the forerunner cells (fo), also begins to express cas. At $90 \%$ epiboly, expression is seen in all endodermal and forerunner cells and resembles sox17 expression. (I) cas expression in presumptive forerunner cells. $(J, K)$ By $60 \%$ epiboly, forerunners, which continue to express cas, form a compact group of cells that do not involute and stay at the vegetal tip of the axial mesoderm throughout gastrulation. $(L, M)$ Forerunners show strong

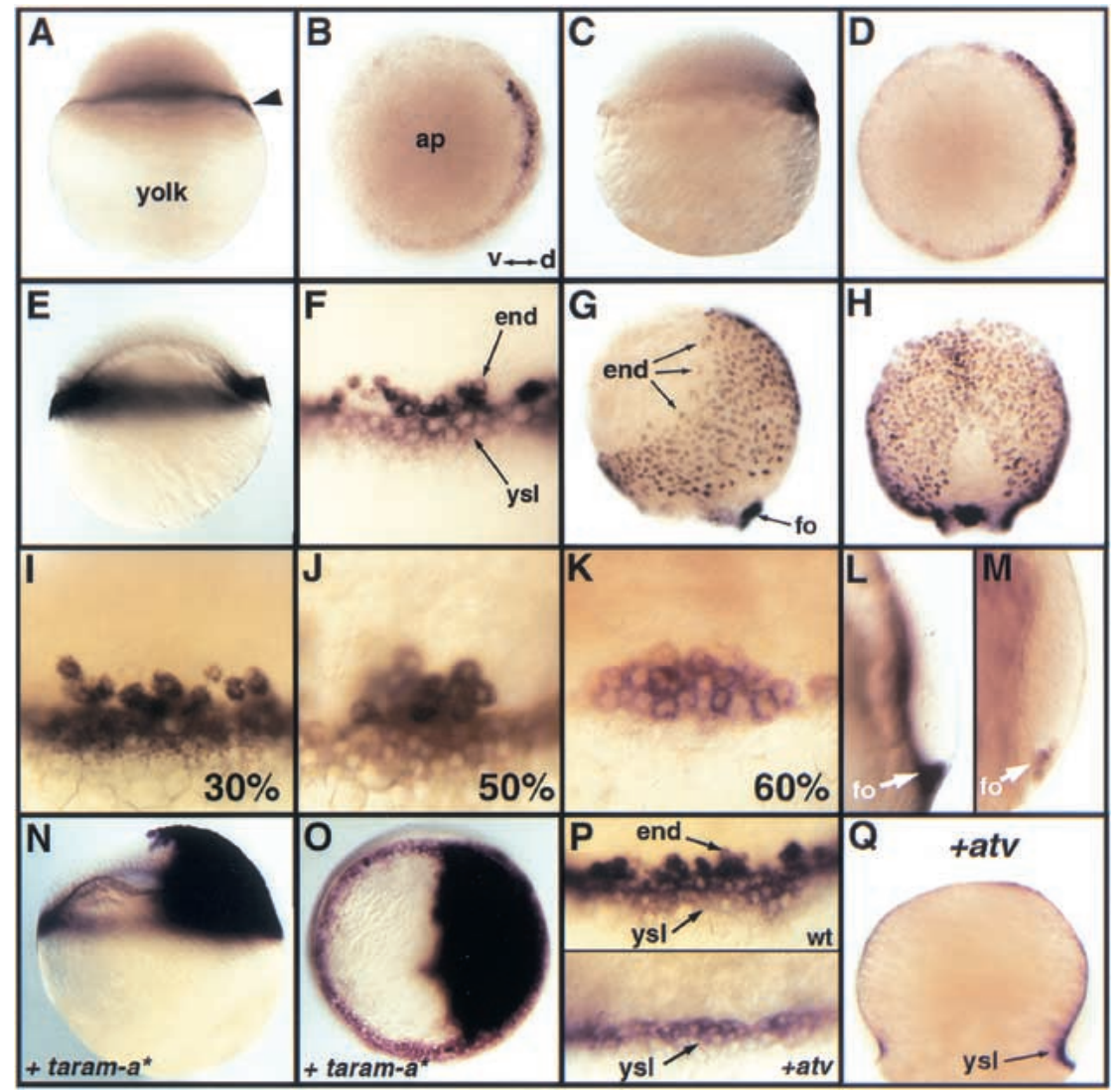

cas expression $(L)$ as well as $s q t(M)$ expression throughout gastrulation. $(N, O)$ Injection of $100 \mathrm{pg}$ of taram- $a^{\star}$ mRNA into one cell at the two-cell stage led to a high level of cas expression in the injected half of the embryo. $(P, Q)$ Injection of a low dose of antivin $(a t v)$ mRNA $(25 \mathrm{pg})$ at the one-cell stage led to the disappearance of embryonic cas expression, whereas the YSL expression appeared unaffected. High magnification views at $40 \%$ epiboly are shown in $P$. The optical cross section in $Q$ shows cas expression present in the YSL, but absent in the endoderm of atv injected embryos at $90 \%$ epiboly. ap, animal pole; d, dorsal; end, endoderm; fo, forerunner cells; v, ventral; wt, wild type; ysl, yolk syncytial layer.

pression. To distinguish between these models, we tested whether bon or fau/gata5 could induce cas expression in cells that do not normally express cas. Wild-type embryos were injected with bon, fau/gata5, or both at the 1-4-cell stage and examined for cas expression at $40 \%$ epiboly. Although endodermal cas expression at the margin was somewhat increased, presumably reflecting increased endoderm formation, no cas expression was observed outside the margin (Fig. 5D-F; data not shown), showing that bon or fau/gata5, alone or in combination, is not sufficient to activate cas expression in nonmarginal cells.

Figure 3. cas expression in cas, bon, and fau/ gata5 mutant embryos. Animal pole views with dorsal to the right at shield stage $(6 \mathrm{~h}$ postfertilization [hpf]). $(A-C)$, and dorsal views with anterior to the top at $80 \%$ epiboly $(8.3 \mathrm{hpf} ; D-F)$. $(A, D)$ In cas mutants, no cas expression is observed in the embryo proper, whereas cas appears to be up-regulated in the yolk syncytial layer (YSL). $(B, E)$ In bon mutants, cas expression is observed in a few endodermal cells (arrows), the forerunner cells (arrowheads), and the YSL. $(C, F)$ In fau/gata5 mutants, cas expression in the endodermal cells appears weaker (arrows), whereas cas expression in the forerunner cells (arrowheads) and the YSL appears normal.

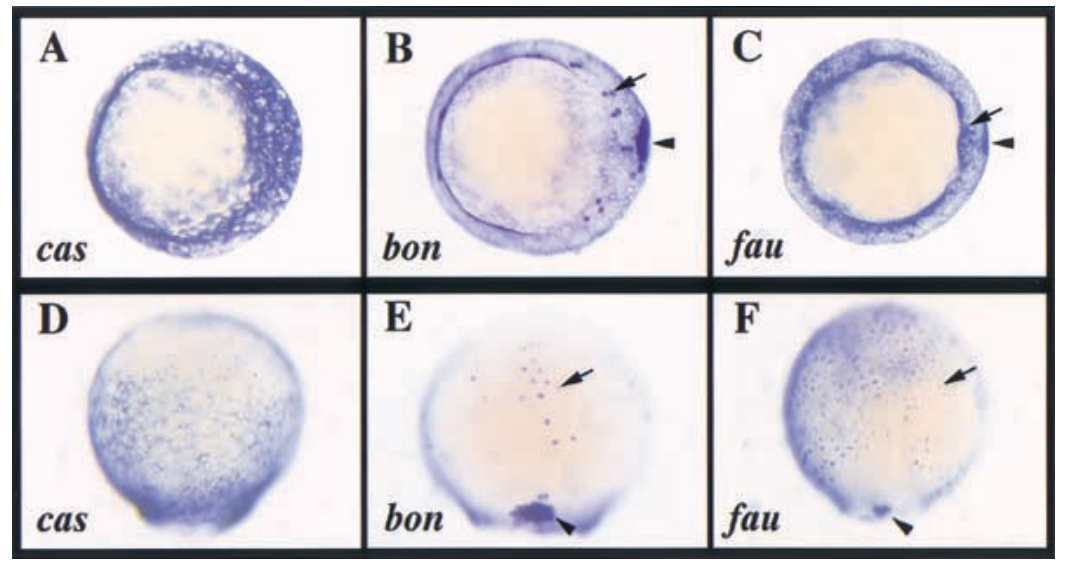




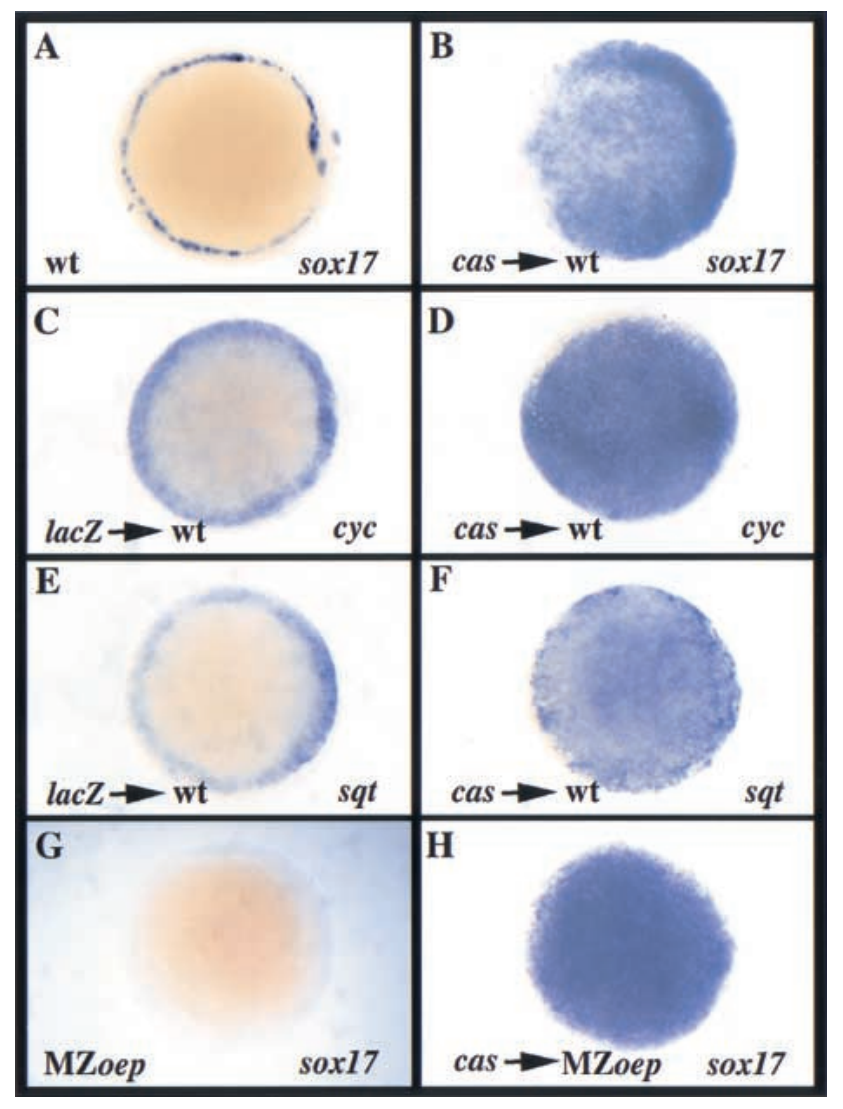

Figure 4. cas overexpression induces $c y c$ and $s q t$ expression in wild-type embryos, and induces sox17 expression in MZoep mutants. $(A, B, G, H)$ Animal pole views at shield stage $(6 \mathrm{~h}$ postfertilization [hpf]) with dorsal to the right $(A) .(C-F)$ Animal pole views at $50 \%$ epiboly $(5.3 \mathrm{hpf})$ with dorsal to the right $(C, E)$. $(A, B)$ A characteristic salt-and-pepper distribution of sox17-expressing endodermal cells is observed throughout the margin in uninjected wild-type embryos $(A)$, whereas injection of cas mRNA at the 1-4-cell stage led to confluent patches of sox17 expression throughout the embryo $(B) .(C-F)$ Injection of cas mRNA at the 1-4-cell stage induced ectopic cyc and sqt expression in wild-type embryos $(D, F) .(G, H)$ Whereas no sox17 expression is observed in uninjected MZoep mutants $(G)$, injection of cas mRNA at the 1-4-cell stage induced strong sox17 expression $(H)$. wt, wild type.-

\section{Ectopic cas expression leads to the transfating of mesodermal cells into endoderm}

cas overexpression leads to widespread sox17 expression, suggesting that cas is able to activate sox 17 expression in nonendodermal cells and possibly direct them to become endoderm. To test this possibility, we ectopically expressed cas and GFP (100 pg each) in single marginal blastomeres at the 16-cell stage and determined the position of the injected cells at shield stage (6 hpf). For example, Figure 6A shows a clone resulting from a dorsal injection. First we examined sox17 expression in the injected embryos at shield stage and found that, in all cases, strong sox17 expression was induced around the labeled clone (Fig. 6B). Next, we examined the fate of dorsal, dorsolateral, lateral, and ventral clones. Examination at 24 hpf showed that the descendants of injected dorsal cells, which would normally populate prechordal plate and notochord, had colonized the entire extent of the gut tube (Fig. 6C). The conversion of dorsal axial mesoderm into endoderm led this embryo (shown in Fig. 6C) to be cyclopic and exhibit U-shaped somites (Fig. $6 \mathrm{D}, \mathrm{E})$. Of 106 embryos injected dorsally, cyclopia was seen in $44(41.5 \%)$ and U-shaped somites in $68(64.1 \%)$. We further characterized the loss of anterior axial mesoderm in dorsally injected embryos by examining the expression of $g s c$, a prechordal plate marker, and hgg1, a hatching gland marker (Thisse et al. 1994), at 50\% and $80 \%$ epiboly, respectively: of 74 embryos injected dorsally, we found a complete absence of gsc expression in 4 $(5.4 \%)$, and a strong reduction, such as that seen in Figure $6 \mathrm{G}$, in $54(73 \%)$; of 80 embryos injected dorsally, we found a complete absence of $h g g 1$ in $16(20 \%)$, and a strong reduction, such as seen in Figure 6I, in $56(70 \%)$. To examine the axial mesoderm in dorsally injected embryos, we analyzed shh expression at $24 \mathrm{hpf}$, which, at this stage, is present in the notochord, floor plate, and part of the diencephalon (Krauss et al. 1993). Of $76 \mathrm{em}-$ bryos injected dorsally, we found a complete absence of shh expression in $4(5.2 \%)$, and patchy expression, such as that seen in Figure $6 \mathrm{~K}$, in $68(89.5 \%)$.

Embryos injected dorsolaterally with cas and GFP also exhibited a decrease in notochordal tissue, as evidenced by loss of shh expression and kinking of the embryos (data not shown). Laterally injected embryos showed a unilateral loss of somitic muscle with a concomitant enlargement of fox $A 2$ expression on the cas-injected side (data not shown). Embryos injected ventrally with cas and GFP exhibited an accumulation of GFP-expressing cells in the tail, which displayed an abnormal shape characterized by a large, empty ventral blister, instead of forming ventral mesenchyme (Fig. 6L). To analyze the fate of the cas-expressing ventral cells, which would normally give rise to blood, we examined the expression of the carbonic anhydrase gene (C. Thisse and B. Thisse, unpubl.), which is specifically expressed in blood cells and the otic vesicle (Fig. 6M). Of 81 embryos injected ventrally, we observed a total absence of carbonic anhydrase expression in blood tissue in $18(22 \%)$, and a partial reduction, such as that seen in Figure $6 \mathrm{~N}$, in $48(59.3 \%)$, whereas the otic vesicle expression was unaffected.

Figure 6O-R show our interpretation of these data by representing the fate maps of a noninjected embryo (Fig. 6O), dorsally injected embryos (a smaller clone in Fig. 6P and a larger clone in Fig. 6Q), and a ventrally injected embryo (Fig. 6R). cas misexpression in a clone of cells located centrally within the embryonic shield at the onset of gastrulation resulted in the transfating of the prechordal plate progenitors into endoderm, as revealed partly by the loss of $g s c$ and $h g g 1$ expression (Fig. 6G,I,P). cas misexpression in a larger dorsal clone affected both anterior and posterior axial mesoderm, resulting in embryos that lack both prechordal and notochordal tissues (Fig. 6K,Q). cas misexpression in ventral clones resulted in embryos that lacked nearly all blood cells, but whose 
Figure 5. cas overexpression induces ectopic sox17 expression in bon and fau/gata5 mutant embryos, whereas bon and faul gata5 cannot induce ectopic cas expression in wild-type embryos. Animal pole views at $6 \mathrm{~h}$ postfertilization $(\mathrm{hpf}, A-C)$ and $40 \%$ epiboly stage (5 hpf; $D-F)$. (A-C) Injection of cas mRNA at the 1-4-cell stage expanded sox17 expression to a comparable extent in wild-type, bon, and fau/gata5 mutant embryos. $(D-F)$ Injection of bon or fau/gata5 mRNA in wild-type embryos was not able to induce ectopic cas expression away from the margin.

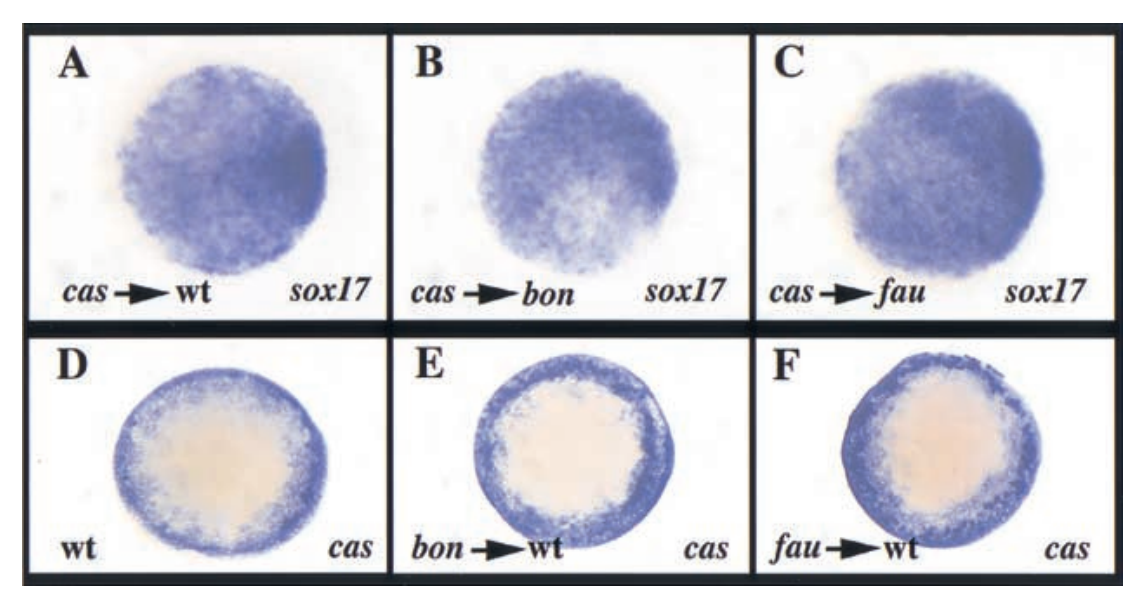

other mesodermal derivatives appeared unaffected (Fig. $6 \mathrm{~N}, \mathrm{R})$.

cas misexpression at the animal pole at the 256-cell stage (Fig. 6S,T), a territory normally fated to become epidermis, telencephalon, and eye, led to ectopic sox17 expression (Fig. 6W), but did not affect the morphology of the injected embryos (Fig. 6U). Injections performed at earlier developmental stages (32-128-cell stage) gave essentially the same results. However, due to the extensive spreading and mixing of cells derived from these earlier stage blastomeres, some of them reached the margin during the blastula stage and became incorporated in the endoderm. Localized injections in single animal pole blastomeres at the 256-cell stage gave rise to smaller clones of cells that remained in the epiblast; at $24 \mathrm{hpf}$, labeled cells appeared in various ectodermal locations, sometimes within differentiated structures such as the neural tube, and often on top of the yolk (Fig. 6V). Nevertheless, disappearance of ectodermal derivatives, indicative of transfating, was never observed. One possible explanation for these observations is the finding that cas misexpression in the animal pole does not induce ectopic sqt expression in the embryo proper, but only in a few cells of the enveloping layer (evl; Fig. 6X), whereas cas misexpression in the margin induces strong sqt expression in embryonic cells (Fig. 6Y). In conditions in which Nodal activity is inhibited by Antivin, injection of cas in a marginal blastomere at the 16-cell stage resulted in the induction of sqt only in evl cells (Fig. 6Z), demonstrating that the ability of cas to induce sqt expression in the embryo proper is dependent on Nodal signaling. The finding that sqt is not induced in the embryo proper by cas misexpression at the animal pole may similarly be explained by the fact that this region has previously been shown to be a ground state for the Activin/Nodal signaling pathway (Thisse et al. 2000).

\section{Discussion}

cas encodes a novel member of the SoxF group of HMG domain transcription factors

We have provided genetic and molecular evidence that cas encodes a novel member of the SoxF group of tran- scription factors. Sox17, whose Xenopus orthologs can activate endodermal gene expression in animal caps (Hudson et al. 1997), also belongs to this group, but several points argue against Cas and XSox17 being equivalent. First, sequence analyses suggest that cas is a novel SoxF member and not a recently duplicated version of zebrafish sox17. Second, cas but not Xsox17 can restore sox17 expression in cas mutants (J. Alexander and D.Y.R. Stainier, unpubl.), indicating a clear functional difference between them.

In both zebrafish and Xenopus, Gata5 and a Mix-type protein (Bon in zebrafish and Mixer in Xenopus) have been shown to regulate sox17 expression (Henry and Melton 1998; Alexander and Stainier 1999; Kikuchi et al. 2000; Reiter et al. 2001; Xanthos et al. 2001). And based on the temporal characteristics of the Xsox17 expression pattern, an additional upstream regulator probably initiates its expression in Xenopus (Xanthos et al. 2001). It is thus possible that, much like Cas regulates sox17 expression in zebrafish, another member of the SoxF group regulates Xsox17 expression in Xenopus.

The conservation seen in the pathways responsible for endoderm formation in zebrafish and Xenopus suggest that similar factors may be involved in this process in mammals. Although mouse Sox18 does not appear to be expressed in the developing endoderm (Pennisi et al. 2000 ), it will be interesting to investigate the early expression of Sox 7 and Sox17 in the mouse embryo, as well as any possible roles they may play in endoderm formation.

\section{cas may regulate sox17 directly}

cas is initially expressed in the YSL and later appears in marginal blastomeres. The embryonic expression of cas is clearly regulated by Nodal signaling, as shown by its absence in antivin-injected embryos and MZoep mutants. Unlike previously identified genes, cas expression in late blastula-stage embryos may be restricted to the endodermal progenitors; cas is expressed in a subset of fau/gata5-expressing cells, appearing more spottily distributed throughout the margin (Fig. 2F,P). sox17 expression appears to be activated in all cas-expressing cells, 


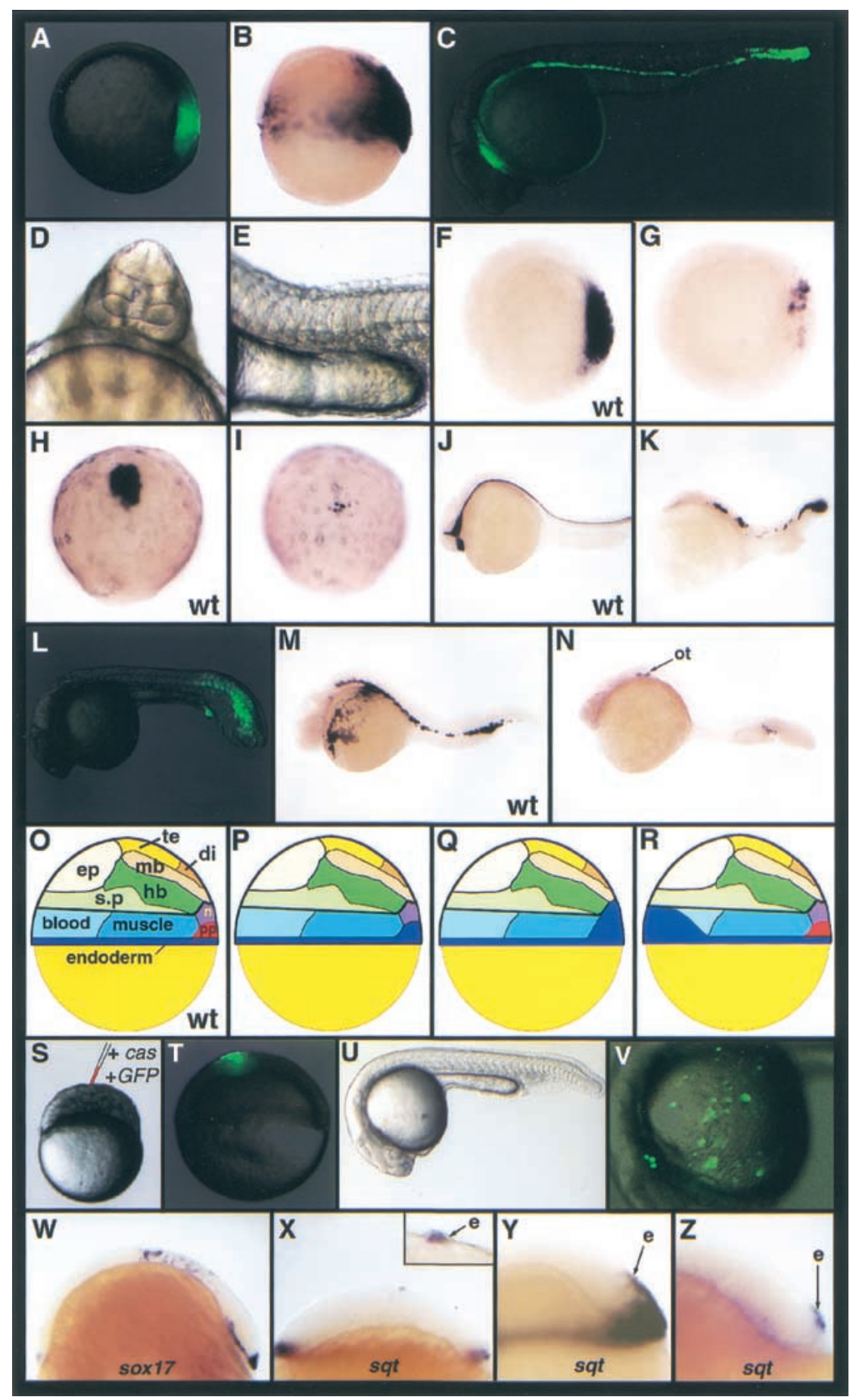

Figure 6. Misexpression of cas results in the transfating of presumptive mesodermal cells into endoderm. GFP fluorescence at shield stage (6 hours postfertilization [hpf]; $A$; animal pole view; $T$; lateral view), and at $24 \mathrm{hpf}(C, L, V$; lateral views). Lateral view with dorsal to the right of sox17 expression at shield stage $(B, W)$. gsc expression at $50 \%$ epiboly (5.3 hpf; F,G; animal pole views, dorsal to the right). hgg1 expression at $80 \%$ epiboly (8.3 hpf; $H, I$; dorsal views, anterior to the top). shh expression at $24 \mathrm{hpf}\left(J, K_{\text {; }}\right.$ lateral views). carbonic anhydrase expression at $24 \mathrm{hpf}(M, N$; lateral views). Fate map, as adapted from Thisse et al. (2000) of uninjected $(O)$ and cas-injected embryos $(P-R)$ at the onset of gastrulation (5.3 hpf). sqt expression at $30 \%$ epiboly $(4.7 \mathrm{hpf} ; X)$ and $40 \%$ epiboly (5 hpf; $Y, Z$ ); lateral views, dorsal to the right. (A) Coinjection of $100 \mathrm{pg}$ of cas mRNA and $100 \mathrm{pg}$ of GFP mRNA into a marginal blastomere at the 16-cell stage gives rise to marginal clones of cells which were screened at the onset of gastrulation to determine their localization along the dorso-ventral axis. A dorsal clone is shown here. (B) Embryo with a labeled dorsal clone was examined for sox17 expression, which was strongly induced dorsally. $(C)$ Embryo shown in $A$ at 24 hpf. GFP-expressing cells accumulate in the entire endoderm, from the level of the pharyngeal arches anteriorly to the anal opening in the posterior trunk region. More caudally, GFP-expressing cells are observed underlying the notochord in the anterior part of the tail, and in the notochord and tail bud more posteriorly. $(D)$ High-magnification view of the head of the embryo shown in $C$, revealing a cyclopic phenotype. $(E)$ The same embryo exhibits Ushaped somites, indicative of a reduction in axial mesoderm. $(F, G)$ gsc expression in a dorsally injected embryo $(G)$ is severely reduced as compared to wild type $(F)$. $(H, I)$ Expression of $h g g 1$, a prechordal plate marker, in a dorsally-injected embryo $(I)$ is nearly abolished as compared to wild type $(H)$. $(J, K)$ shh expression in a dorsally-injected embryos $(K)$ is severely reduced as compared to wild type $(J) .(L)$ Embryo with a labeled ventral clone at 24 hpf. GFP-expressing cells accumulate in the tail, which displays an abnormal shape characterized by a large, empty ventral blister instead of ventral mesenchyme. $(M, N)$ The blood expression of carbonic anhydrase has almost completely disappeared in a ventrally-injected embryo, whereas the otic vesicle (ot) expression is unaffected. (O) Fate map of a wild-type embryo at the onset of gastrulation (di, diencephalon; ep, epidermis; hb, hindbrain; mb, midbrain; $\mathrm{n}$, notochord; pp, prechordal plate; sp, spinal chord; te, telencephalon). $(P \mid$ Misexpression of cas in a clone of cells located in the central part of the embryonic shield results in the transfating of the prechordal plate into endoderm, resulting in the loss of $g s c(G)$ and $h g g 1(I)$ expression. (Q) A larger dorsal clone affects both anterior and posterior axial mesoderm, resulting in embryos that lack both prechordal and notochordal mesoderm, as shown in $K$. $(R)$ Ventral clones result in embryos that lack almost all blood cells but appear unaffected in the other mesodermal derivatives, as shown in $N$. (S,T) An embryo injected with cas and GFP mRNA in an animal pole blastomere at the 256-cell stage $(S)$ is shown at shield stage in $T$. (U,V) At 24 hpf, embryos injected at the animal pole have a wild-type morphology $(U)$ and the GFP-expressing cells do not populate the endoderm but are often found on top of the yolk $(V) .(W, X)$ Injection of cas at the animal pole induces sox $17(W)$ but not sqt $(X)$ expression in the embryo proper. In such embryos, sqt expression is only induced in a few cells of the enveloping layer (evl cells; e), an extraembryonic structure. $(Y, Z)$ In contrast, marginal cas injection induces strong sqt expression in embryonic cells around the margin $(Y)$ and this induction is blocked by Atv overexpression ( $Z$; this embryo was injected with $25 \mathrm{pg}$ of atv at the two-cell stage and with cas at the 16-cell stage. In such embryos, sqt expression is only induced in a few evl cells). wt, wild type.- 
except the YSL, starting $\sim 80 \mathrm{~min}$ after the onset of cas expression. cas overexpression can induce sox17 expression in MZoep mutants as well as in antivin-injected embryos (C. Thisse and B. Thisse, unpubl.), indicating that cas can regulate sox17 expression independently of Nodal signaling. The regulation of endodermal sox17 expression by Cas is cell autonomous as shown by cell transplantation experiments (Alexander et al. 1999), as well as by the finding that cas overexpression in the YSL, in contrast to cas overexpression throughout the embryo, does not up-regulate sox17 expression (Y. Kikuchi and D.Y.R. Stainier, unpubl.). In addition, HMG domainbinding sites are present upstream of sox17 (Y. Kikuchi and D.Y.R. Stainier, unpubl.), suggesting that the regulation of sox17 by Cas may be direct.

\section{Potential roles for cas in the YSL}

cas is first expressed in the dorsal YSL at high stage, which coincides with the initial expression of sqt (Feldman et al. 1998; Rebagliati et al. 1998a; Sampath et al. 1998). cas and sqt expression subsequently spread around the margin and by $30 \%$ epiboly cas, cyc, and sqt expression are evident all around the margin (Erter et al. 1998; Rebagliati et al. 1998b). This coexpression in the margin and the regulation of embryonic cas expression by Nodal signaling led us to further examine their relationship; although cas expression in the YSL is clearly independent of Nodal signaling, interestingly, overexpression of cas throughout the embryo led to widespread cyc and sqt expression. (These data are in contrast to the inability of bon, which is also initially expressed in the dorsal YSL [Alexander et al. 1999], to induce cyc and sqt expression [Kikuchi et al. 2000].) Apart from the forerunner cell phenotype, cyc and sqt expression appear normal in cas mutants, leaving the physiological role of cas in $c y c$ and sqt expression unclear. It may be that defects in cyc and sqt expression in cas mutants are too subtle to detect by in situ, that other regulators of $c y c$ and $s q t$ expression are able to compensate for the lack of Cas function, or that Cas normally regulates sqt expression only in the forerunners.

It is interesting to note that cas mutants, like cyc mutants, exhibit left-right asymmetry defects (Liang et al. 2000); gene expression that is left-sided in the anterior lateral plate mesoderm (LPM) and diencephalon of wildtype embryos becomes randomized in cas and cyc mutants (Rebagliati et al. 1998b; Sampath et al. 1998; Liang et al. 2000). In thinking about the basis for the laterality defects in cas mutants, it is useful to consider bon mutants, in which, despite a nearly complete absence of endoderm, left-right asymmetry appears normal (J. Alexander and D.Y.R. Stainier, unpubl.). Another difference between cas and bon mutants is the formation of forerunner cells, which is defective in cas mutants (Alexander et al. 1999) but apparently normal in bon mutants (Kikuchi et al. 2000). Thus, the laterality defects observed in cas mutants are unlikely to be due to the absence of endoderm but, rather, a consequence of either the lack of forerunner cells or the lack of cas function in the YSL. Given the physical proximity of the YSL to the diencephalon during gastrulation (Woo and Fraser 1995) and to the anterior LPM during mid- to late-segmentation stages (Stainier et al. 1993), it is possible that cas function in the YSL influences left-right asymmetry. Alternatively, given the fact that both cas and sqt are expressed in the forerunner cells, it is possible that these cells are involved in setting up left-right asymmetry. The endocytic properties of forerunner cells /Cooper and D'Amico 1996) could in fact be a key part of this function and lead to differential signaling on the left and right sides of the embryo. It will be interesting to investigate further whether either or both of these tissues are involved in setting up left-right asymmetry as well as whether cas regulates left-right asymmetry by regulating $c y c$ and/or sqt expression or through some other mechanism.

The finding that cas is expressed in the YSL also suggests an alternative hypothesis for the mesodermal defects observed in cas mutants. These defects include the failure of the bilateral myocardial populations to migrate and fuse at the midline (cardia bifida), as well as similar defects in the vasculature, blood, and pronephros (Alexander et al. 1999). We originally proposed that the absence of endoderm may underlie these defects (Alexander et al. 1999). It may be, however, that defects in the YSL, which is also closely apposed to several mesodermal tissues in mid- to late-segmentation stages, may cause the mesodermal defects seen in cas mutants, or that the YSL and endoderm both contribute to the migration of mesodermal tissues. Tissue specific loss-offunction analyses will be required to further investigate the basis for these mesodermal defects.

\section{cas, bon and fau/gata5}

cas, bon and fau/gata5 encode transcription factors that directly or indirectly regulate sox17 expression. These three genes exhibit a similar pattern of expression; all initiate expression at approximately the same time in the YSL and the marginal zone of the embryo. In the margin bon expression is the broadest (Alexander at al. 1999) and cas the most restricted. We have previously shown that, prior to gastrulation, bon and fau/gata5 expression appear normal in cas, bon and fau/gata5 mutants (Alexander et al. 1999; Reiter et al. 2001), suggesting that Cas function downstream of, or in parallel to, Bon and Fau/Gata5. These data were confirmed by the finding that cas overexpression in MZoep mutants does not induce bon or fau/gata5 expression.

Neither bon nor fau/gata5 overexpression restores sox17 expression in cas mutants (Alexander and Stainier 1999; Reiter et al. 2001), further indicating that cas functions downstream of, or in parallel to, bon and fau/gata5. We confirmed these data by showing that cas overexpression in bon and fau/gata5 mutants led to widespread sox17 expression. To further examine whether Cas acts downstream of, or in parallel to, Bon and Fau/Gata5, we overexpressed bon and fau/gata5 in wild-type embryos and examined cas expression. We found that bon or faul 
gata5, alone or in combination, were not able to induce cas expression in nonmarginal cells. These data are consistent with the observations that when bon and faul gata5 were overexpressed in wild-type embryos, epiboly proceeded normally and no ectopic sox 17 expression was induced (Reiter et al. 2001), in contrast to what was observed in cas-injected embryos. Furthermore, coinjection of bon and fau/gata5 in cyc;sqt double mutants was not able to restore significant sox17 expression (Y. Kikuchi and D.Y.R. Stainier, unpubl.), in contrast to what was observed in cas-injected MZoep mutants. All these data are consistent with at least two different models: cas, bon, and fau/gata5 may be regulated independently by Nodal signaling and function in parallel; alternatively, bon and fau/gata5 may act upstream of cas, with a third factor, $\mathrm{X}$, being restricted to the marginal zone and necessary for cas expression. Some combination of these two models is also possible, and molecular dissection of the cas promoter may help to further investigate this question.

\section{cas is sufficient to convert mesoderm into endoderm}

Ectopic expression of cas in presumptive mesodermal cells leads to their transfating into endoderm. cas-expressing dorsal mesodermal cells lose gsc and hggl expression (Fig. 6G,I), and colonize the entire length of the gut tube (Fig. 6C). Such embryos display cyclopia due to the loss of prechordal plate, and U-shaped somites due to the loss of notochord (Fig. 6D,E). It is interesting to note that cas-expressing cells, unlike taram- $a^{\star}$-expressing cells (Peyriéras et al. 1998), never contribute to the hatching gland. We therefore suggest that this organ is not of endodermal origin as previously suggested (Peyriéras et al. 1998), but, rather, it most likely represents a mesodermal derivative whose formation, similar to that of the endoderm, requires high level, and/or sustained, Nodal signaling.

Ventral mesodermal cells, normally fated to give rise to blood as well as to other tissues, lose their ability to form blood when expressing cas. However, cas expression is not able to redirect their migration, so these cells end up colonizing the posterior gut tube and the tail region (Fig. 6L). Importantly, despite the ability of cas to up-regulate $c y c$ and $s q t$ expression when expressed throughout the embryo, ectopic expression in 1 of 16 blastomeres does not lead to general defects, such as broadened or duplicated axes, indicative of ectopic sqt expression (Erter et al. 1998). Rather, the effects on embryonic development appear to be limited to the casexpressing cells. Altogether, these data indicate that cas is able to initiate an endoderm-specific program in cells not fated to become endoderm. However, this ability appears to be restricted to the mesoderm; when cas is overexpressed in animal pole blastomeres at the 256-cell stage, these cells do not transfate from ectoderm into endoderm. In summary, only when overexpressed near the margin is cas able to transfate cells into endoderm. It will be important to identify the factors responsible for this spatial restriction. In this context, it is interesting to reiterate that cas is unable to induce in $M Z$ Zep mutants strong expression of endodermal markers other than sox17, such as foxA2 and fau/gata5, indicating that other Nodal-regulated components are essential for the full endodermal program. cas misexpression in the animal pole, unlike that in the margin, does not lead to ectopic nodal gene expression, offering one possible explanation for the inability of these cells to transfate into endoderm.

\section{Conclusions}

Phenotypic analyses have shown that cas is the central regulator of sox17 expression and endoderm formation in zebrafish (Alexander et al. 1999; Alexander and Stainier 1999; Reiter et al. 2001). The isolation of cas, analysis of its expression pattern, and gain-of-function experiments lead us to propose the following model: cas expression in the YSL initiates independently of Nodal signaling. At late blastula stages, Nodal signaling induces bon, faul gata5, and cas expression in marginal blastomeres. Bon, Fau/Gata5, and Cas directly or indirectly regulate sox17 expression in the forming endodermal cells, which also continue to express fau/gata5 and cas during gastrulation. Several important issues remain to be addressed, in particular the differential regulation of bon, fau/gata5, and cas expression in the margin. These genes may become activated at different thresholds of Nodal signaling, and/or incorporate inputs from other marginally localized factors acting autonomously or nonautonomously. Resolution of this issue, along with the identification and analyses of additional regulators of endoderm formation, should further our understanding of the molecular events that direct individual marginal blastomeres toward an endodermal fate in vertebrate embryos.

\section{Materials and methods}

\section{Zebrafish strains}

Adult zebrafish and embryos were maintained and staged as described (Westerfield 1995). Heterozygous carriers were identified by random intercrosses and intercrossed to obtain mutant embryos. Adult MZoep ${ }^{m 134}$ fish were a generous gift of Will Talbot (Stanford University). The following mutant alleles were used: bon $^{s 9}$ (Kikuchi et al. 2000), faut ${ }^{\text {tm236a }}$ (Chen et al. 1996; Reiter et al. 1999), cas $^{\text {ta56 }}$ (Chen et al. 1996), and cas $^{\text {s4 }}$.

\section{Isolation of the CG569 gene}

A full-length clone was isolated from a gastrula cDNA library (gift of Thierry Lepage) inserted between XhoI and EcoRI of pBluescript IISK ${ }^{+}$. The GenBank accession number for cas is AF362749.

\section{Wholemount in situ hybridization}

For CG569/cas probe synthesis, NotI was used to linearize the plasmid and T7 RNA polymerase to generate antisense RNA. Other riboprobes were prepared according to published instructions. In situ hybridizations shown in Figure 2 and Figure 6 were performed as described by Thisse and Thisse (http://www- 
igbmc.u-strasbg.fr/zf_info/zbook/chapt9/9.82.html); the others as described in Alexander et al. (1998).

Genetic mapping and genomic sequencing of $\operatorname{cas}^{\text {ta56 }}$ and $\operatorname{cas}^{\text {s4 }}$

CG569 was mapped on the Goodfellow zebrafish radiation hybrid panel (Research Genetics) using the following PCR primers: 5'-CCAGATTGCTGCTGATTTTG-3' and 5'-TCAATACAAA CTGGTCAAGTTAACAAC-3'.

To extract genomic DNA from single embryos, $30 \mathrm{hpf}$ embryos were digested overnight in $10 \mathrm{mM}$ Tris- $\mathrm{HCl} \mathrm{pH} 7.5,1 \mathrm{mM}$ EDTA, $0.1 \%$ SDS, $100 \mathrm{mM} \mathrm{NaCl}, 20 \mu \mathrm{g} / \mathrm{mL}$ proteinase $\mathrm{K}$ at $55^{\circ} \mathrm{C}$. Mutant genomic DNA was purified from ten $30 \mathrm{hpf}$ embryos using genomic DNA extraction kits (Scotlab Bioscience). To sequence the genomic locus, 4 primer pairs were designed based on CG569 sequence data. The PCR fragments were cloned into the pGEM-T vector (Promega). We performed three independent PCR reactions for each primer pair, picked two independent clones from each PCR reaction, and sequenced them using an ABI 377 DNA sequencer.

\section{Linkage analysis}

CG569 genomic fragments were amplified with PCR primers (5'-CTGGCAGATAAGCGTCCGTACATGC-3' and 5' -CTGA GTAAGCAGACCGTGGCAGATGC-3'). PCR conditions were $94^{\circ} \mathrm{C}$ for $5 \mathrm{~min}(1 \mathrm{cycle})$, then $94^{\circ} \mathrm{C}$ for $30 \mathrm{sec}, 63^{\circ} \mathrm{C}$ for $30 \mathrm{sec}$ and $72^{\circ} \mathrm{C}$ for $1 \mathrm{~min}\left(40\right.$ cycles), followed by $72^{\circ} \mathrm{C}$ for $5 \mathrm{~min}(1$ cycle). Amplified products were digested with $B f a \mathrm{I}$ for $\mathrm{Cas}^{\text {ta56 }}$ or RsaI for $\mathrm{Cas}^{\mathrm{s}}$, and resolved on $2 \%$ agarose gels. Following in situ hybridization, embryos were genotyped as described above.

\section{$m R N A$ injections}

To introduce the s4-type point mutation into a cas injection vector, we performed site-directed mutagenesis using QuickChange $^{\mathrm{TM}}$ Site-Directed Mutagenesis Kit (Stratagene) according to the manufacturer's instruction using the primer pair $\left(5^{\prime}\right.$ GCAGAAAGACTACGGATCCAGCG ${ }^{\star}$ TACCATTGACTATC CTAACTAC-3' and $5^{\prime}$-GTAGTTAGGATAGTCAATGGTA $\mathrm{C}^{\star}$ GCTGGATCCGTAGTCTTTCTGC- ${ }^{\prime}$; ${ }^{*}$ indicates the site of the mutation) and $\mathrm{pCS}^{+}$-cas template. The coding region of $\mathrm{cas}^{\mathrm{s} 4}$ was verified by sequencing both strands. Capped mRNA encoding cas or $\operatorname{cas}^{s 4}$ was transcribed in vitro from pCS2 ${ }^{+}$-cas or pCS2 ${ }^{+}{\text {- } a s^{s 4}}^{\text {s }}$, using the SP6 mMESSAGE mMACHINE system (Ambion). Capped antivin, taram- $a^{\star}$, bon, fau/gata5, lacZ, and gfp mRNA were prepared according to published instructions (Peyriéras et al. 1998; Thisse et al. 1999; Kikuchi et al. 2000; Reiter et al. 2000). Embryos were injected with the following mRNAs: antivin (25 pg), taram- $a^{\star}(100 \mathrm{pg})$, bon $(100 \mathrm{pg})$, cas $(100 \mathrm{pg})$, fau/gata5 (60 pg), lacZ (100 pg). Embryos injected at the 1-4-cell stage with cas mRNA fail to undergo epiboly and do not exhibit a clear dorsal axis; they were fixed for in situ at $6 \mathrm{hpf}$, which is equivalent to shield stage. For the transfating experiments, 100 pg each of cas and $g f p$ mRNA were coinjected into a single marginal blastomere of 16-cell stage embryos, or with a $120 \mathrm{ng} / \mu \mathrm{L}$ solution of cas and GFP mRNA in an animal pole blastomere at the 256-cell stage.

\section{Acknowledgments}

We thank Sarah Howard and Alex Navarro for outstanding technical assistance; Will Talbot for MZoep fish; Peter Koopman for expert help with sequence and clustering analyses; Don Kane for unpublished information about the LG7 centromere; Hiroyuki Takeda and Takuya Sakaguchi for open discussions about unpublished data; Janet Heasman for discussions about endoderm formation; Leonard $\mathrm{D}^{\prime}$ Amico for discussions about forerunner cells; Jeremy Reiter, Le Trinh, Elke Ober, and Heather Verkade for critical comments on the manuscript. Y.K. was funded by a long-term fellowship from the Human Frontier Science Program and is now an American Heart Association Postdoctoral Fellow. A.A is funded by the ministère de l'Education Nationale et de la Recherche. J.A. was supported by an American Heart Association Predoctoral Fellowship and D.Y. by an Amgen fellowship of the Life Sciences Research Foundation and a Career Award in the Biomedical Sciences from the Burroughs Wellcome Fund. This work was supported in part by funds from the Institut National de la Santé et de la Recherche Médicale, the Centre National de la Recherche Scientifique, the Hôpital Universitaire de Strasbourg, the Association pour la Recherche sur le Cancer and the Ligue Nationale Contre le Cancer to B.T. and C.T., and from the American Heart Association, the Packard Foundation, the Life and Health Insurance Medical Research Fund and the NIH (DK58181) to D.Y.R.S

The publication costs of this article were defrayed in part by payment of page charges. This article must therefore be hereby marked "advertisement" in accordance with 18 USC section 1734 solely to indicate this fact.

\section{References}

Alexander, J. and Stainier, D.Y.R. 1999. A molecular pathway leading to endoderm formation in zebrafish. Curr. Biol. 9: $1147-1157$.

Alexander, J., Stainier, D.Y.R., and Yelon, D. 1998. Screening mosaic F1 females for mutations affecting zebrafish heart induction and patterning. Dev. Genet. 22: 288-299.

Alexander, J., Rothenberg, M., Henry, G.L., and Stainier, D.Y.R. 1999. casanova plays an early and essential role in endoderm formation in zebrafish. Dev. Biol. 215: 343-357.

Bowles, J., Schepers, G., and Koopman, P. 2000. Phylogeny of the SOX family of developmental transcription factors based on sequence and structural indicators. Dev. Biol. 227: 239255.

Chen, J.N., Haffter, P., Odenthal, J., Vogelsang, E., Brand, M., van Eeden, F.J.M., Furutani-Seiki, M., Granato, M., Hammerschmidt, M., Heisenberg, C.P., et al. 1996. Mutations affecting the cardiovascular system and other internal organs in zebrafish. Development 123: 293-302.

Conlon, F.L., Lyons, K.M., Takaesu, N., Barth, K.S., Kispert, A., Herrmann, B., and Robertson, E.J. 1994. A primary requirement for nodal in the formation and maintenance of the primitive streak in the mouse. Development 120: 19191928.

Cooper, M.S. and D'Amico, L.A. 1996. A cluster of noninvoluting endocytic cells at the margin of the zebrafish blastoderm marks the site of embryonic shield formation. Dev. Biol. 180: 184-198.

Erter, C.E., Solnica-Krezel, L., and Wright, C.V. 1998. Zebrafish nodal-related 2 encodes an early mesendodermal inducer signaling from the extraembryonic yolk syncytial layer. Dev. Biol. 204: 361-372.

Feldman, B., Gates, M.A., Egan, E.S., Dougan, S.T., Rennebeck, G., Sirotkin, H.I., Schier, A.F., and Talbot, W.S. 1998. Zebrafish organizer development and germ-layer formation require nodal-related signals. Nature 395: 181-185.

Gritsman, K., Zhang, J., Cheng, S., Heckscher, E., Talbot, W.S., 
and Schier, A.F. 1999. The EGF-CFC protein one-eyed pinhead is essential for nodal signaling. Cell 97: 121-132.

Henry, G.L. and Melton, D.A. 1998. Mixer, a homeobox gene required for endoderm development. Science 281: 91-96.

Hudson, C., Clements, D., Friday, R.V., Stott, D., and Woodland, H.R. 1997. Xsox $17 \alpha$ and $-\beta$ mediate endoderm formation in Xenopus. Cell 91: 397-405.

Johnson, S.L., Africa, D., Horne, S., and Postlethwait, J.H. 1995. Half-tetrad analysis in zebrafish: Mapping the ros mutation and the centromere of linkage group I. Genetics 139: 17271735.

Kaestner, K.H., Knöchel, W., and Martinez, D.E. 2000. Unified nomenclature for the winged helix/forkhead transcription factors. Genes \& Dev. 14: 142-146.

Kikuchi, Y., Trinh, L.A., Reiter, J.F., Alexander, J., Yelon, D., and Stainier, D.Y.R. 2000. The zebrafish bonnie and clyde gene encodes a Mix family homeodomain protein that regulates the generation of endodermal precursors. Genes \& Dev. 14: 1279-1289.

Krauss, S., Concordet, J.P., and Ingham, P.W. 1993. A functionally conserved homolog of the Drosophila segment polarity gene $h h$ is expressed in tissues with polarizing activity in zebrafish embryos. Cell 75: 1431-1444.

Kwok, C., Critcher, R., and Schmitt, K. 1999. Construction and characterization of zebrafish whole genome radiation hybrids. In Methods in Cell Biology. The Zebrafish: Genetics and genomics (eds. H.W. Detrich, M. Westerfield, and L.I. Zon), pp. 287-302. Academic Press, San Diego, CA.

Liang, J.O., Etheridge, A., Hantsoo, L., Rubinstein, A.L., Nowak, S.J., Izpisua Belmonte, J.C., and Halpern, M.E. 2000. Asymmetric nodal signaling in the zebrafish diencephalon positions the pineal organ. Development 127: 5101-5112.

Osada, S.I. and Wright, C.V. 1999. Xenopus nodal-related signaling is essential for mesendodermal patterning during early embryogenesis. Development 126: 3229-3240.

Payne, T.L., Postlethwait, J.H., and Yelick, P.C. 2001. Functional characterization and genetic mapping of alk8. Mech. Dev. 100: 275-289.

Pennisi, D., Gardner, J., Chambers, D., Hosking, B., Peters, J., Muscat, G., Abbott, C., and Koopman, P. 2000. Mutations in Sox18 underlie cardiovascular and hair follicle defects in ragged mice. Nat. Genet. 24: 434-437.

Peyriéras, N., Strähle, U., and Rosa, F. 1998. Conversion of zebrafish blastomeres to an endodermal fate by TGF- $\beta$ - related signalling. Curr. Biol. 8: 783-786.

Rebagliati, M.R., Toyama, R., Fricke, C., Haffter, P., and Dawid, I.B. 1998a. Zebrafish nodal-related genes are implicated in axial patterning and establishing left-right asymmetry. Dev. Biol. 199: 261-272.

Rebagliati, M.R., Toyama, R., Haffter, P., and Dawid, I.B. 1998b. cyclops encodes a nodal-related factor involved in midline signaling. Proc. Natl. Acad. Sci. 95: 9932-9937.

Reiter, J.F., Alexander, J., Rodaway, A., Yelon, D., Patient, R., Holder, N., and Stainier, D.Y.R. 1999. Gata5 is required for the development of the heart and endoderm in zebrafish. Genes \& Dev. 13: 2983-2995.

Reiter, J.F., Kikuchi, Y., and Stainier, D.Y.R. 2001. Multiple roles for Gata5 in zebrafish endoderm formation. Development 128: 125-135.

Renucci, A., Lemarchandel, V., and Rosa, F. 1996. An activated form of type I serine/threonine kinase receptor TARAM-A reveals a specific signalling pathway involved in fish headorganiser formation. Development 122: 3735-3743.

Rodaway, A., Takeda, H., Koshida, S., Broadbent, J., Price, B., Smith, J.C., Patient, R., and Holder, N. 1999. Induction of the mesendoderm in the zebrafish germ ring by yolk cellderived TGF- $\beta$ family signals and discrimination of mesoderm and endoderm by FGF. Development 126: 3067-3078.

Saitou, N. and Nei, M. 1987. The neighbor-joining method: A new method for reconstructing phylogenetic trees. Mol. Biol. Evol. 4: 406-425.

Sampath, K., Rubinstein, A.L., Cheng, A.M., Liang, J.O., Fekany, K., Solnica-Krezel, L., Korzh, V., Halpern, M.E., and Wright, C.V. 1998. Induction of the zebrafish ventral brain and floorplate requires cyclops/nodal signalling. Nature 395: 185-189.

Schier, A.F., Neuhauss, S.C., Helde, K.A., Talbot, W.S., and Driever, W. 1997. The one-eyed pinhead gene functions in mesoderm and endoderm formation in zebrafish and interacts with no tail. Development 124: 327-342.

Stainier, D.Y., Lee, R.K., and Fishman, M.C. 1993. Cardiovascular development in the zebrafish. I. Myocardial fate map and heart tube formation. Development 119: 31-40.

Strähle, U., Blader, P., Henrique, D., and Ingham, P.W. 1993. Axial, a zebrafish gene expressed along the developing body axis, shows altered expression in cyclops mutant embryos. Genes \& Dev. 7: 1436-1446.

Thisse, C., Thisse, B., Halpern, M.E., and Postlethwait, J.H. 1994. goosecoid expression in neurectoderm and mesendoderm is disrupted in zebrafish cyclops gastrulas. Dev. Biol. 164: 420-429.

Thisse, C. and Thisse, B. 1999. Antivin, a novel and divergent member of the TGF $\beta$ superfamily, negatively regulates mesoderm induction. Development 126: 229-240.

Thisse, B., Wright, C.V., and Thisse, C. 2000. Activin- and Nodal-related factors control antero-posterior patterning of the zebrafish embryo. Nature 403: 425-428.

Tremblay, K.D., Hoodless, P.A., Bikoff, E.K., and Robertson, E.J. 2000. Formation of the definitive endoderm in mouse is a Smad2-dependent process. Development 127: 3079-3090.

Warga, R.M. and Kimmel, C.B. 1990. Cell movements during epiboly and gastrulation in zebrafish. Development 108: 569-580.

Warga, R.M. and Nüsslein-Volhard, C. 1999. Origin and development of the zebrafish endoderm. Development 126: 827838.

Weber, H., Symes, C.E., Walmsley, M.E., Rodaway, A.R., and Patient, R.K. 2000. A role for GATA5 in Xenopus endoderm specification. Development 127: 4345-4360.

Werner, M.H., Huth, J.R., Gronenborn, A.M., and Clore, G.M. 1995. Molecular basis of human 46X,Y sex reversal revealed from the three-dimensional solution structure of the human SRY-DNA complex. Cell 81: 705-714.

Westerfield, M. 1995. The Zebrafish book: A guide for the laboratory use of zebrafish. University of Oregon Press, Eugene, OR.

Woo, K. and Fraser, S.E. 1995. Order and coherence in the fate map of the zebrafish nervous system. Development 121: 2595-2609.

Xanthos, J.B., Kofron, M., Wylie, C., and Heasman, J. 2001. Maternal VegT is the initiator of a molecular network specifying endoderm in Xenopus laevis. Development 128: 167180.

Zaret, K. 1999. Developmental competence of the gut endoderm: Genetic potentiation by GATA and HNF3/fork head proteins. Dev. Biol. 209: 1-10.

Zhang, J., Talbot, W.S., and Schier, A.F. 1998. Positional cloning identifies zebrafish one-eyed pinhead as a permissive EGFrelated ligand required during gastrulation. Cell 92: 241-251. 


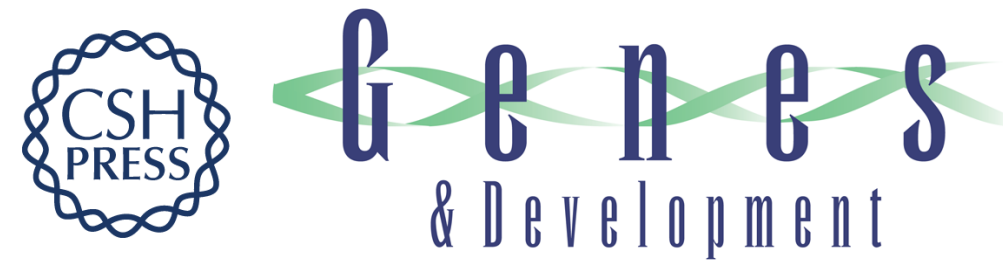

\section{casanova encodes a novel Sox-related protein necessary and sufficient for early endoderm formation in zebrafish}

Yutaka Kikuchi, Antoine Agathon, Jonathan Alexander, et al.

Genes Dev. 2001, 15:

Access the most recent version at doi:10.1101/gad.892301

References

This article cites 44 articles, 23 of which can be accessed free at: http://genesdev.cshlp.org/content/15/12/1493.full.html\#ref-list-1

License

Email Alerting

Receive free email alerts when new articles cite this article - sign up in the box at the top Service right corner of the article or click here.

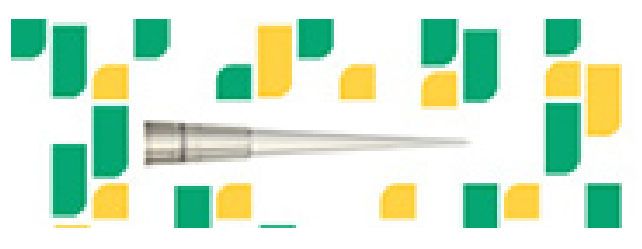

Focused on your science. 